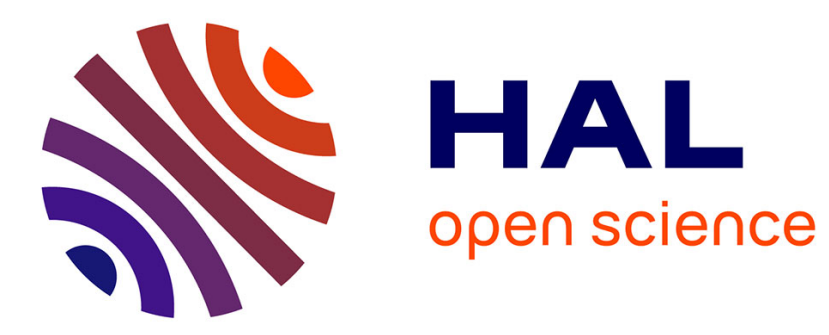

\title{
Theoretical study of polyiodide formation and stability on monolayer and bilayer graphene
}

\author{
Damien Tristant, Pascal Puech, I.C. Gerber
}

\section{To cite this version:}

Damien Tristant, Pascal Puech, I.C. Gerber. Theoretical study of polyiodide formation and stability on monolayer and bilayer graphene. Physical Chemistry Chemical Physics, 2015, 17 (44), pp.3004530051. 10.1039/c5cp04594k . hal-01756983

\section{HAL Id: hal-01756983 \\ https://hal.science/hal-01756983}

Submitted on 30 Jan 2019

HAL is a multi-disciplinary open access archive for the deposit and dissemination of scientific research documents, whether they are published or not. The documents may come from teaching and research institutions in France or abroad, or from public or private research centers.
L'archive ouverte pluridisciplinaire HAL, est destinée au dépôt et à la diffusion de documents scientifiques de niveau recherche, publiés ou non, émanant des établissements d'enseignement et de recherche français ou étrangers, des laboratoires publics ou privés. 


\title{
Theoretical study of polyiodide formation and stability on monolayer and bilayer graphene ${ }^{\dagger}$
}

\author{
Damien Tristant, ${ }^{a, b}$ Pascal Puech, ${ }^{b}$ and Iann C. Gerber* ${ }^{* a}$
}

\author{
Received Xth $X X X X X X X X X X 20 X X$, Accepted Xth $X X X X X X X X X 20 X X$ \\ First published on the web Xth $X X X X X X X X X X 20 X X$ \\ DOI: 10.1039/b000000x
}

The presence of polyiodide complexes have reported several times when carbon-based materials have been doped by iodine molecules, but their formation mechanism remains unclear. By using first-principles calculations that include nonlocal correlation effects by means of van der Waals density functional approach, we propose that the formation of triiodide $\left(\mathrm{I}_{3}^{-}\right)$and pentaiodide $\left(\mathrm{I}_{5}^{-}\right)$is due to a large density of iodine molecules $\left(\mathrm{I}_{2}\right)$ in interaction with carbonaceous substrate. As soon as the concentration of surface iodine reaches the threshold value of $12.5 \%$ for a graphene monolayer and $6.25 \%$ for a bilayer, these complexes spontaneously appear. The corresponding structural, energetic aspects, electronic structures and vibrational frequencies support this statement. An upshift of the Dirac point from the Fermi level with values of 0.45 and $0.52 \mathrm{eV}$ is observed for adsorbed complexes on graphene and intercalated complexes between two layers, respectively. For doped-graphene, it corresponds to a graphene hole density to be around $1.1 \times 10^{13} \mathrm{~cm}^{-2}$, in quantitative agreement with experiments. Additionally, we have studied the thermal stability at room temperature of these adsorbed ions on graphene by means of ab initio molecular dynamics, also showing a successful p-doping with polyiodide complexes.

\section{Introduction}

Graphene, which belongs to the family of carbon nanoscale structure has attracted the attention of researches due to interesting physical and chemical properties. These intrinsic electronic properties and their tunings when molecules are adsorbed, makes this material a suitable candidate for improving electronic components. Theoretical and experimental studies have demonstrated that the deposition of halogen molecules modulates the electronic structure of graphene layer ${ }^{1-15}$ in a completely reversible process. Specially, adsorption of iodine molecules $\mathrm{I}_{2}$ is intriguing because of its intrinsic capability to exist with different forms, ${ }^{16}$ with a wide variety of chemical properties. Among experimental works, Raman spectroscopy indicates a signature of iodide anion formations, which can be assigned to the formation of triiodide $\left(\mathrm{I}_{3}^{-}\right)$and pentaiodide $\left(\mathrm{I}_{5}^{-}\right)$, when graphene layer is exposed to iodine molecules. ${ }^{14,17,18}$ Moreover, iodide anions are also present in iodine-doped bilayer $^{19}$, carbon nanotubes (CNTs) and organic polymers. ${ }^{20-25}$ It is believed that adsorbed iodine molecules generate iodide

$0 \dagger$ Electronic Supplementary Information (ESI) available: [details of any supplementary information available should be included here]. See DOI: 10.1039/b000000x/

$0^{a}$ Université de Toulouse; INSA, UPS, CNRS; LPCNO, 135 avenue de Rangueil, F-31077 Toulouse, France. Fax: (+33) 05615596 97; Tel: (+33) 0561559645

$0^{b}$ CEMES, UPR 8011, CNRS-Université de Toulouse, 29 rue Jeanne Marvig, BP 94347, 31055 Toulouse, France.

0*E-mail: igerber@insa-toulouse.fr ions through charge transfer process from structures composed of carbon atoms, but the origin of the polyiodide complex formation remains unknown. This electronic transfer allows to provide a negative charge to iodine molecules, which avoids the creation of cation $\mathrm{I}^{+}$.

In this theoretical work using density functional theory (DFT), taking into account for weak intermolecular forces, we first show that monolayer and bilayer of graphene can be efficiently doped by polyiodide ions $\mathrm{I}_{3}^{-}$and $\mathrm{I}_{5}^{-}$. We also demonstrate that the formation of these complexes is due to an agglomeration of iodine molecules on a graphene sheet, which can also be considered as a reasonable model of large CNT. The thermal stability of the adsorbed ions is analyzed from $a b$ initio molecular dynamics (AIMD), carried out at $300 \mathrm{~K}$. We present our investigations of structural, energetic properties, electronic structure and vibrational frequencies of iodine species adsorbed and intercalated on graphene monolayer and bilayer respectively. Computational details are described in the following section. Our results of isolated and adsorbed iodine species are then reported. We can clearly attribute vibrational modes and their corresponding frequencies to the presence of $\mathrm{I}_{2}, \mathrm{I}_{3}^{-}$and $\mathrm{I}_{5}^{-}$. Interestingly we prove that the formation of these complexes is due to the either a high concentration or a strong confinement of $\mathrm{I}_{2}$ undergoing strong electronic transfer from graphene-systems. In fact, the areal hole density of the graphene monolayer is about $1.1 \times 10^{13} \mathrm{~cm}^{-2}$. Simulation of the frequency and energetic properties of adsorbed $\mathrm{I}_{2}$ as a function of iodine concentration adsorbed on graphene and interca- 
lated between two layers show respectively a threshold value, $12.5 \%$ and $6.25 \%$, where the phase transition of diatomic iodine molecules to complexes takes place. We conclude, in section 5, that the polyiodide formation still exists at $300 \mathrm{~K}$ and adsorbed species lead to a power spectrum shift of the Gpeak, around $3 \mathrm{~cm}^{-1}$ indicating successful p-doping with iodine species at $300 \mathrm{~K}$.

\section{Computational details}

For the carbon structures, we use a lattice constant of graphene equal to $a_{0}=2.46 \AA$, close to the experimentally obtained value $(2.459 \AA)$ for a graphite at low temperatures. ${ }^{26} \mathrm{We}$ also add a vacuum region of $20 \AA$ to avoid interaction between periodic images in the $z$ direction, since the stability of the work function values is insured as soon as the distance between two layers is $17.8 \AA$. For the bilayer systems, a vacuum of around $22 \AA$

We have also studied a bilayer structure containing 4 carbon atoms in its primitive cell with an interlayer distance of 3.33 $\AA$ in good agreement with experimentally obtained value. ${ }^{27}$ In order to determine the effects of polyiodide creation, several hexagonal cells containing from 32 to 100 carbon atoms in interaction with 5 iodine molecules are considered. So we use $(4 \times 4)$ to $(5 \times 5)$ primitive cells of carbon structures, where for example, $(4 \times 4)$ corresponds to $15.6 \%\left(\mathrm{C}_{32} 5 \mathrm{I}_{2}\right.$ for graphene $)$ and $7.8 \%\left(\mathrm{C}_{64} 5 \mathrm{I}_{2}\right.$ for bilayer $)$ iodine concentration. In the following, we investigate three isolated iodine molecules, $\mathrm{I}_{2}$ and linear $\mathrm{I}_{3}^{-}$are each simulated in a $36 \times 36 \times 36 \AA^{3}$ box and angled $\mathrm{I}_{5}^{-}$located in a $50 \times 50 \times 50 \AA^{3}$ box. To identify vibrational modes of the adsorbed complexes, we study triiodide and pentaiodide interactions with a graphene layer containing $(6 \times 6)$ and $(7 \times 7)$ primitive cells respectively, as illustrated in Figure 1 .

Using density functional theory (DFT), the structural and electronic properties of iodine-graphene and bilayer systems are obtained with the Vienna $a b$ initio simulation package (VASP). ${ }^{28-31}$ Ions cores are modeled with projector augmented wave (PAW) pseudopotentials. ${ }^{32}$ The $2 s$ and $2 p$ states of carbon, $5 s$ and $5 p$ states of iodine are treated explicitly in the valence. The plane-wave basis set cutoff energy is set to 400 $\mathrm{eV}$ with a Gaussian smearing method ${ }^{33}$ of $0.005 \mathrm{eV}$ width, in order to assure well converged total energy and force values. To include van der Waals interactions in our calculations, we use the optB86b-vdW scheme. ${ }^{34,35}$ This particular choice of exchange-correlation functional is based on previous works. ${ }^{10,36-39}$. For the sake of comparisons, we have also computed the Tkatchenko-Scheffler (TS) method ${ }^{40,41}$ on the adsorption of a single iodine molecule which is an interesting test case for describing the nonlocal anisotropic polarization. vdWTS-GGA (PBE functional) predicts the same geometry properties and adsorption energy than the optB86b-vdW method, see
Table 1 from the Electronic Supporting Information (ESI). This comparison strongly stress the importance of the many body van der Waals interactions that stem from the strong anisotropic polarization of low-dimensional materials, as the TS method is based on a pair-wise approximation.

All the atoms are allowed to relax until the maximum of all forces acting on them became smaller than $0.01 \mathrm{eV} . \AA^{-1}$. In order to determine the distortion effect of graphene layer potentially induced by the iodine adsorption, the cell shape of the polyiodide-graphene structure, Figure 2(a), was also allowed to relax but at constant volume, see the ESI for the comparison. Since no drastic changes have been observed, we have kept fixed the calculation cell dimensions for the rest of the presented results. The k-point sampling is always based on a $\Gamma$-centered grid for all types of calculations. We use, to optimize the structures, a single k-point for iodine molecules, and a $(9 \times 9 \times 1)$ grid for $(4 \times 4)$ primitive cells of graphene up to a $(5 \times 5 \times 1)$ grid for the largest graphene and bilayer supercells. For the density of states (DOS) calculations, the tetrahedron integration method with Blöchl corrections ${ }^{42}$ is used, with a $(29 \times 29 \times 1)$ grid for the $(4 \times 4)$ graphene supercell and a $(23 \times 23 \times 1)$ grid for the $(4 \times 4)$ bilayer supercell. Similar grids are used to determine charge transfer and band structures for graphene and bilayer systems. Bader charge analysis is carried out using Henkelman's group program, ${ }^{43-45}$ and visualized with VESTA software. ${ }^{46}$

We can compare the energy stability of two structures via the adsorption energy $E_{\text {ad }}$ of $n$ iodine molecules on the system which means graphene or bilayer. This energy can be calculated from the following equation :

$$
E_{\mathrm{ad}}=\frac{1}{n}\left(E_{n \mathrm{I}_{2} @ \text { system }}-E_{\mathrm{system}}-n E_{\mathrm{I}_{2}}\right),
$$

where $E_{n \mathrm{I}_{2} @ \text { system }}$ is the total energy of doped system with $n$ iodine molecules, $E_{\mathrm{I}_{2}}$ is the energy of single free doping iodine molecule and $E_{\text {system }}$ is the total energy of the pristine graphene or bilayer.

Based on the linear dispersion of the band structure near the Dirac point, the areal hole density of doped graphene can be estimated using the following equation:

$$
N_{h}=\frac{1}{\pi\left(\hbar v_{F}\right)^{2}}\left|E_{F}-E_{D}\right|^{2},
$$

where $N_{h}$ is hole areal density, $v_{F}=1.1 \times 10^{6} \mathrm{~m} / \mathrm{s}$ is the Fermi velocity of graphene, extracted from our calculation on the graphene primitive cell, consistent with experimental values ${ }^{47}$. $E_{F}$ and $E_{D}$ are the energy position of the Fermi level and of the Dirac point respectively.

The $a b$ initio molecular dynamics simulations were carried out using VASP. A time step $\Delta t$ of 0.5 fs was chosen and the temperature was set to $300 \mathrm{~K}$ using a Nosé Hoover chain thermostat. ${ }^{48-50}$ Starting from optimized geometries of 
the graphene and graphene-doped systems respectively, $10 \mathrm{ps}$ were performed. To be sure that the system is properly thermalized, we use in our calculations the last $5 \mathrm{ps}$. One of the ways to calculate a vibrational spectrum from ab initio molecular dynamics trajectory is the power spectrum $P(\omega)$, depending on the nuclear velocities $v=\mathrm{d} r_{n} / \mathrm{d} t$, where $r_{n}$ describes the position of the $n^{\text {th }}$ atom. To approximate the analytical time derivative, we use centered-difference quotient method at 7 points. For each atom, the autocorrelation of the velocity is expressed as $\langle v(\tau) v(t+\tau)\rangle_{n}$ and the sum of all the correlation functions of particles is Fourier transformed to obtain the power spectrum of the system ${ }^{51}$ according to

$$
P(\omega)=m \int_{-\infty}^{+\infty}\langle v(\tau) v(t+\tau)\rangle e^{-i \omega t} \mathrm{~d} t,
$$

where $m$ is the reduced mass of the system and $\tau$ equals $5 \mathrm{ps}$.

\section{Geometric analysis of single and adsorbed polyio- dides}

Like all of the other halogens, iodine occurs naturally as a diatomic molecule $\mathrm{I}_{2}$, in solid or gaseous form, but it can form a series of anions, that consist of chains of iodines with one or more extra electrons. The two most common of them are the linear species $\mathrm{I}_{3}^{-}$and the angled species $\mathrm{I}_{5}^{-}$, see Figure 2 of the ESI. In Table 2 of the ESI, we summarize the main geometric parameters and the vibrational modes after geometry optimizations of isolated diatomic iodine, triiodide and pentaiodide ions. They are compared with theoretical methods as the multireference configuration interaction (MRCI) ${ }^{16}$ and also experimental data. Generally our method using nonlocal functional, optB86b-vdW gives I-I bond length values and stretching mode values in good agreement with the experiment and theoretical results. We have identified the vibrational modes of $\mathrm{I}_{3}^{-}$and $\mathrm{I}_{5}^{-}$ions, as summarized in Table 1. Symmetrical vibrational frequencies of diatomic iodine molecules, with in-plan or perpendicular orientation are 200 and $180 \mathrm{~cm}^{-1}$ respectively, as extracted from a previous work. ${ }^{10}$ Although these molecules are physisorbed, vibrational frequencies are lower than the values belonging to isolated molecules. In addition, when the molecule is in-plan orientation, the theoretical value is in agreement with the experimental value of $180 \mathrm{~cm}^{-1}$. The adsorption height between the ions and the average height of the graphene layer, $3.69 \AA$ and $3.65 \AA$ for $\mathrm{I}_{3}^{-}$and $\mathrm{I}_{5}^{-}$respectively, suggests a physisorption effect. Their vibrational frequencies correspond to the isolated ion values, leading to an underestimation of the symmetric stretching mode value of $\mathrm{I}_{3}^{-}$, while the theoretical value of $\mathrm{I}_{5}^{-}$is in perfect agreement with the experimental value of $154 \mathrm{~cm}^{-1}$.

In order to understand the formation of polyiodides on graphene layer, we have studied two nanoscale systems, com-

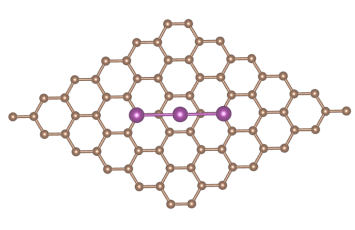

(a)

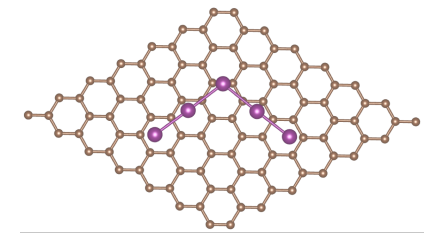

(b)
Fig. 1 (Color online) View of different adsorption sites of molecules (purple circles) on graphene layer (brown circles) : (a) triiodide and (b) pentaiodide adsorbed on $(6 \times 6)$ and $(7 \times 7)$ primitive cells of graphene layer respectively.

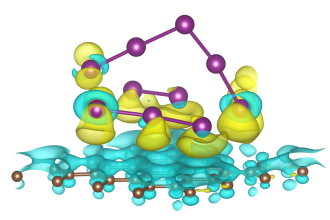

(a)

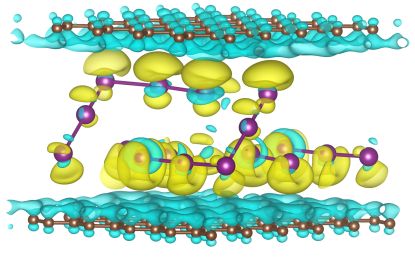

(b)
Fig. 2 (Color online) Charge density difference showing electron transfer between iodine molecules and $(4 \times 4)$ graphene layer $(a)$ and $(4 \times 4)$ bilayer $(b)$. Yellow represents positive electronic densities which accept electrons and cyan stands for negative densities which donate electrons.

posed of five iodine molecules. Figure 2 shows these systems after geometry optimization : (a) the agglomeration of five iodine molecules on a small graphene surface, means 15.6 $\%$ of iodine concentration; (b) the confinement of these particles between two graphene layers, means $7.8 \%$ of iodine concentration. Table 2 summarizes the energetic and geometric properties corresponding to the adsorption of $\mathrm{I}_{2}, \mathrm{I}_{3}^{\delta-}$ and $\mathrm{I}_{5}^{\delta-}$ molecules, with a partial charge $\delta$, at various iodine concentrations up to $15.6 \%$, since below this value the lateral interactions between iodine molecules are less important, due to large $\mathrm{I}_{2}-\mathrm{I}_{2}$ distances.

At high iodine concentration (15.6\% for graphene system and $7.8 \%$ for bilayer system), it is clear that triiodide and pentaiodide species are predominant in these systems. The interatomic distances of $\mathrm{I}_{2}$ is increased by $0.1 \AA$, while the $\mathrm{I} \cdot \cdot \mathrm{I}$ bond length and angle values of complexes are similar to those summarized in Table 1 and Table 2 of the ESI. At low iodine concentration (10.0\% for graphene system and $5.0 \%$ for bilayer system) the molecules remain diatomics, with I-I bond length value around $2.70 \AA$. Between these two concentrations, at $12.5 \%$ for the graphene system and $6.25 \%$ for the bilayer system, the molecular nature is not clearly defined, and oscillates between $\mathrm{I}_{2}$ and $\mathrm{I}_{5}^{\delta-}$ states. Therefore, $12.5 \%$ and 6.25 $\%$ are the limit value for graphene and bilayer systems respec- 
Table 1 Variation of the bond distance between two iodine atoms $(\AA)$, of the angle between three iodine atoms $\left(^{\circ}\right)$, of the adsorption height between the molecules and the average height of the graphene layer $(\AA)$ and of the vibrational frequencies $v\left(\mathrm{~cm}^{-1}\right)$ for symmetric and anti-symmetric stretching modes of adsorbed $\mathrm{I}_{2}, \mathrm{I}_{3}^{\delta-}$ and $\mathrm{I}_{5}^{\delta-}$. They are compared with experimental $(\mathrm{R}=\mathrm{Raman})$ and other calculated values.

\begin{tabular}{llllll}
\hline Structure & I.・I & Angle & I-graphene & $v$ & \\
\cline { 5 - 6 } Fig.1 & & & & Sym. & A.Sym. \\
\hline (a) & 2.96 & 180 & 3.69 & $106,117(\mathrm{R})^{14}$ & 138 \\
(b) & {$[2.86 ; 3.05]$} & {$[103 ; 181]$} & 3.65 & $154,154(\mathrm{R}){ }^{14}$ & 140 \\
\hline
\end{tabular}

Table 2 Variation of the adsorption energy $\mathrm{E}_{a d}(\mathrm{eV})$, of the bond distance between two iodine atoms $(\AA)$ and the angle between three iodine atoms $\left(^{\circ}\right)$ of $\mathrm{I}_{2}, \mathrm{I}_{3}^{\delta-}$ and $\mathrm{I}_{5}^{\delta-}$, for various concentrations (\%), deposited on graphene monolayer and between two layers.

\begin{tabular}{|c|c|c|c|c|c|c|c|c|}
\hline \multirow{2}{*}{$\begin{array}{l}\text { Structure } \\
\text { Fig. } 2\end{array}$} & \multirow{2}{*}{$\begin{array}{l}\mathrm{I}_{2} \\
\text { concentration }\end{array}$} & \multirow[t]{2}{*}{$\mathrm{E}_{a d}\left(\mathrm{I}_{2}\right)$} & \multicolumn{4}{|l|}{ I-I } & \multicolumn{2}{|c|}{ Angle } \\
\hline & & & $\mathrm{I} \cdot \cdot \mathrm{I}$ & $\mathrm{I}_{2}$ & $\mathrm{I}_{3}^{\delta-}$ & $\mathrm{I}_{5}^{\delta-}$ & $\mathrm{I}_{3}^{\delta-}$ & $\mathrm{I}_{5}^{\delta-}$ \\
\hline \multirow[t]{3}{*}{ (a) } & 15.6 & -0.83 & 2.78 & & {$[2.83 ; 3.06]$} & {$[2.86 ; 3.07]$} & 171 & {$[97 ; 177]$} \\
\hline & 12.5 & -0.69 & & 2.71 & & {$[2.80 ; 3.30]$} & & {$[85 ; 172]$} \\
\hline & 10.0 & -0.50 & & 2.69 & & & & \\
\hline \multirow[t]{3}{*}{ (b) } & 7.8 & -0.59 & 2.80 & & {$[2.93 ; 2.89]$} & {$[2.86 ; 3.02]$} & 177 & {$[85 ; 176]$} \\
\hline & 6.2 & -0.49 & & 2.71 & & {$[2.78 ; 3.20]$} & & {$[85 ; 177]$} \\
\hline & 5.0 & -0.21 & & 2.70 & & & & \\
\hline
\end{tabular}

tively, where the phase transition of iodine diatomic molecules to complexes takes place. A high iodine concentration or a confinement implies an increase in the adsorption energy, which means that the creation of new interatomic bonds improves the system stability, see Table 2 .

\section{Analysis of vibrational frequency and electronic structure of polyiodide complexes}

To support our arguments on the polyiodide formation, we calculated also the vibrational frequencies and the effective charges of molecules at $0 \mathrm{~K}$, summarized in Table 3 . For each system, the vibrational frequency values obtained from calculations have been compared and classified, according to the stretching modes (symmetric and anti-symmetric) of the isolated and adsorbed molecules $\mathrm{I}_{2}, \mathrm{I}_{3}^{-}$and $\mathrm{I}_{5}^{-}$, see Table 1 and Table 2 of the ESI. At high iodine concentration, both systems have vibrational frequency values around $106 \mathrm{~cm}^{-1}$ and 142 $\mathrm{cm}^{-1}$, related to symmetrical and anti-symmetrical modes of $\mathrm{I}_{3}^{\delta-}$ respectively, while the vibrational frequency values around $155 \mathrm{~cm}^{-1}$ and $145 \mathrm{~cm}^{-1}$ correspond to symmetrical and antisymmetrical modes of $\mathrm{I}_{5}^{\delta-}$ respectively. At this stage, no vibrational frequency related to the diatomic molecule appears. The decrease iodine concentration up to $12.5 \%$ for graphene and $6.25 \%$ for bilayer, brings up a vibrational mode due to stretching of the diatomic molecules at $194 \mathrm{~cm}^{-1}$, while we are witnessing the quenching of vibrational modes from $\mathrm{I}_{3}^{\delta-}$, leaving only the symmetrical stretching of $\mathrm{I}_{2}$ at $159 \mathrm{~cm}^{-1}$. Upon reaching the concentration threshold value, only the vibrational mode of the diatomic iodine appear at $203 \mathrm{~cm}^{-1}$. These results show that the formation of complexes is related to the high con- centration or a confinement of iodine molecules.

The calculations of effective charges show that $\mathrm{I}_{3}^{\delta-}$ and $\mathrm{I}_{5}^{\delta-}$ have a partial charge of -0.10 and -0.06 for the graphene system respectively. In the doped-bilayer system, partial charges increase by 0.19 and 0.25 electron per $\mathrm{I}_{3}^{\delta-}$ and $\mathrm{I}_{5}^{\delta-}$ respectively, compared to the doped-graphene system. Therefore the substrate plays a significant role in complex formation. The addition of a second graphene layer allows the increase of the electronic transfer, see Figure 2.

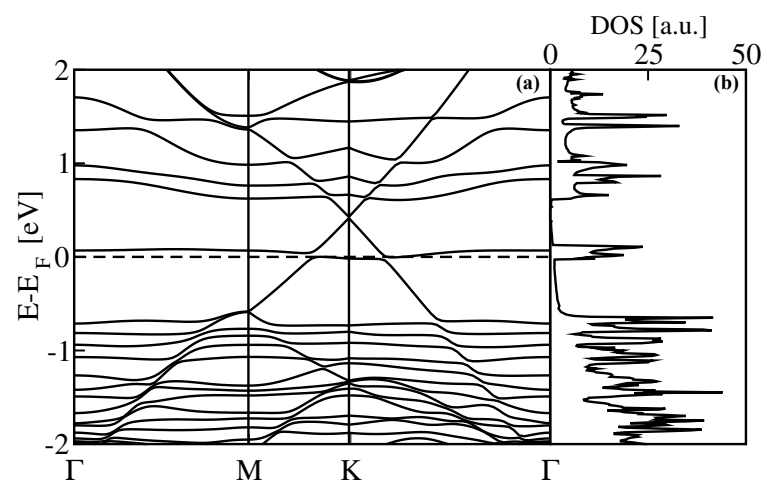

Fig. 3 Band structure (a) and total electronic density of states (b) of halogen complexes adsorbed on $(4 \times 4)$ graphene layer $(15.6 \%$ of iodine concentration). The Fermi level is at zero energy.

The effects of the electron transfer between the two subsystems on the electronic structure properties of the graphene mono- and bilayer are shown in Figures 3 and 4 respectively, for carbon structures containing $(4 \times 4)$ primitive cells. From the band structure in Fig.3(a) and Fig.4(a), it is clear that, the 
Table 3 Variation of the vibrational frequencies $v\left(\mathrm{~cm}^{-1}\right)$ for symmetric and anti-symmetric stretching modes of $\mathrm{I}_{2}, \mathrm{I}_{3}^{\delta-}$ and $\mathrm{I}_{5}^{\delta-}$, of the effective charge of $\mathrm{I}_{3}^{\delta-}$ and $\mathrm{I}_{5}^{\delta-}$ (number of electron), for various concentrations (\%), deposited on graphene monolayer and between two layers.

\begin{tabular}{|c|c|c|c|c|c|c|c|c|}
\hline \multirow{2}{*}{$\begin{array}{l}\text { Structure } \\
\text { Fig. } 2\end{array}$} & \multirow{2}{*}{$\begin{array}{l}\mathrm{I}_{2} \\
\text { concentration }\end{array}$} & \multirow[t]{2}{*}{$v\left(\mathrm{I}_{2}\right)$} & \multicolumn{2}{|c|}{$v\left(\mathrm{I}_{3}^{\delta-}\right)$} & \multicolumn{2}{|c|}{$v\left(\mathrm{I}_{5}^{\delta-}\right)$} & \multirow[t]{2}{*}{$\delta \mathrm{q}\left(\mathrm{I}_{3}^{\delta-}\right)$} & \multirow[t]{2}{*}{$\delta \mathrm{q}\left(\mathrm{I}_{5}^{\delta-}\right)$} \\
\hline & & & Sym. & A.Sym. & Sym. & A.Sym. & & \\
\hline \multirow[t]{3}{*}{ (a) } & 15.6 & & 100 & 142 & 155 & 148 & $\begin{array}{l}-0.10 \\
\end{array}$ & -0.06 \\
\hline & 12.5 & 190 & & & 158 & & & -0.05 \\
\hline & 10.0 & 206 & & & & & & \\
\hline \multirow[t]{3}{*}{ (b) } & 7.8 & & 106 & 133 & 156 & 145 & -0.29 & -0.31 \\
\hline & 6.2 & 194 & & & 159 & & & -0.11 \\
\hline & 5.0 & 203 & & & & & & \\
\hline
\end{tabular}

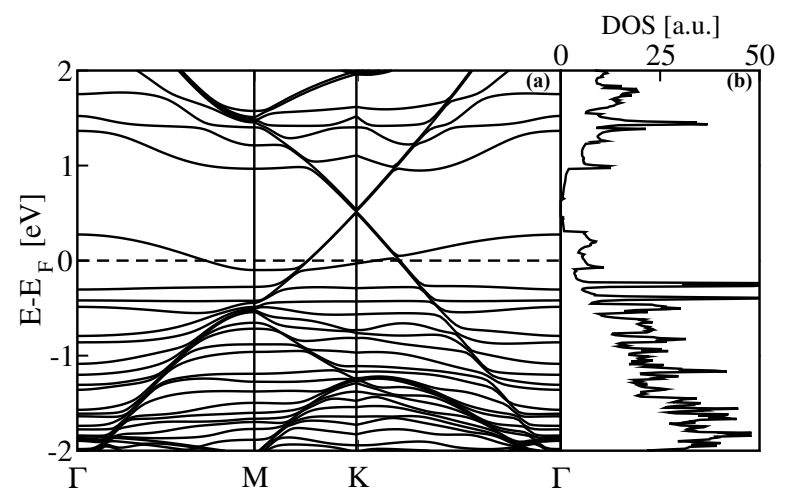

Fig. 4 Band structure (a) and total electronic density of states (b) of $(4 \times 4)$ bilayer graphene intercalated with iodine complexes $(7.8 \%$ of iodine concentration). The Fermi level is at zero energy.

complexes are physisorbed with a weak adsorption energy of $-0.83 \mathrm{eV}$ and $-0.59 \mathrm{eV}$ per diatomic molecules for the dopedgraphene and doped-bilayer respectively. Moreover, the conical crossing band at $\mathrm{K}$ point is still present upon adsorption. The Dirac point is shifted above the Fermi level, $0.45 \mathrm{eV}$ and $0.52 \mathrm{eV}$ for graphene and bilayer systems respectively, corresponding to an electronic transfer from the substrate to the iodine complexes. In order to go further in the interpretation of the electronic band structure, we have also calculated the areal hole density of the graphene system using the equation 2 . It is equal to $1.1 \times 10^{13} \mathrm{~cm}^{-2}$, suggesting an effective hole doping of graphene. This result is consistent with the experimental value $4.4 \times 10^{13} \mathrm{~cm}^{-2}$ obtained in the case of a graphene monolayer doped by a $\mathrm{Br}_{2}$ vapor. ${ }^{11}$ It does suggest that $\mathrm{Br}_{3}^{-}$and $\mathrm{Br}_{5}^{-}$ complexes may indeed adsorbed on the graphene sheet. Our value is much greater than the single adsorbed molecule value of $3.9 \times 10^{11} \mathrm{~cm}^{-2},{ }^{10}$ reflecting the fact that an agglomerate of iodine molecules leads to a larger electron transfer. An impurity band due to the iodine complexes appears just around the Fermi level $\left(E-E_{F}=0\right)$. As shown in Fig.3(b) and Fig.4(b), this resonance located above and below the Fermi level can be attributed to localized p-type orbitals of the halogen complexes, which a predominance of the $\mathrm{p}_{y}$ state for both structures.

\section{Polyiodide molecules adsorbed on graphene at $300 \mathrm{~K}$}

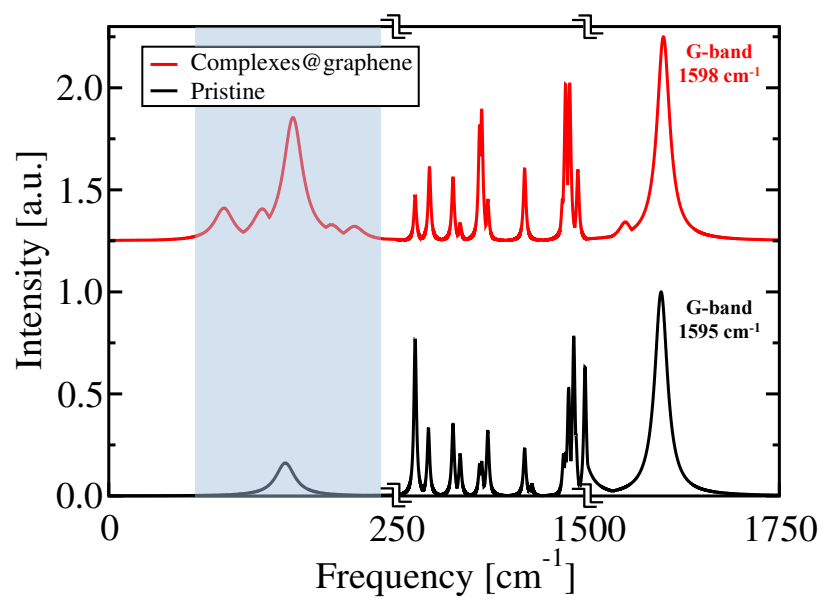

Fig. 5 (Color online) Power spectra at $300 \mathrm{~K}$ of graphene layer (black line) compared to the iodine species adsorbed on the same graphene layer (red line).

The spectra obtained for a pristine and iodine molecules adsorbed on graphene layer at $300 \mathrm{~K}$ are shown in Figure 5. The theoretical curves were fitted to Lorentzian functions, where peak's half-width is set to $10 \mathrm{~K}$. The peak intensities have been normalized relative to the G-band. In these calculations the intensity is not physical. The power spectra shows the G-band peak exactly at the right position. It corresponds to the C-C stretching vibrations from graphene ${ }^{52}$, around $1595 \mathrm{~cm}^{-1}$ for the pristine, and it is slightly shifted by $3 \mathrm{~cm}^{-1}$ for the adsorbed system. This upshift is fully consistent with experimental data ${ }^{7}$ and is quite sensitive to a p-doping effect from graphene layer, that could be modified by the iodine molecule concentration ${ }^{10}$. As non adiabatic effects are not taken explicitly into account, it 
is not possible to determine accurately the Raman shift induced by the iodine doping. Nevertheless, we can take advantage of this to conclude about the sign of the doping. Due to bond length variation, a downshift is associated to n-doping and upshift to p-doping ${ }^{53}$.

In both cases, it can be seen that other features are localized below the G-band frequency. The study of graphene optical characteristic by means of Raman spectrum does not show these vibration modes, ${ }^{15}$ due to the selection rules. A theoretical calculation of Raman spectra can be obtained from the polarizability along the normal mode vectors (not shown here). However, the difference between our graphs leads to the emergence a frequency range $[106 ; 203] \mathrm{cm}^{-1}$ (blue zone), related to vibrational modes of iodine molecules. Using the values summarized in Tables 1 of the ESI and 1, we can identify the vibrational modes in this frequency range. Therefore, $106 \mathrm{~cm}^{-1}$, $160 \mathrm{~cm}^{-1}$ and $203 \mathrm{~cm}^{-1}$, represent the symmetric stretching modes of $\mathrm{I}_{3}^{-}, \mathrm{I}_{5}^{-}$and $\mathrm{I}_{2}$, respectively. The frequency peaks around $140 \mathrm{~cm}^{-1}$, show the presence of anti-symmetric stretching modes of $\mathrm{I}_{3}^{-}$and $\mathrm{I}_{5}^{-}$respectively. The power spectra at 300 $\mathrm{K}$ show the same information as our previous calculations done at $0 \mathrm{~K}$. Moreover, these results are in agreement with experimental data ${ }^{15}$. The agglomeration of iodine particles leads to the formation of complexes.

\section{Conclusion}

In this paper, the study of the polyiodide formation on graphene layer using DFT calculations, including the nonlocal correlation correction, are reported, including the energetic, geometric aspects, as well as the vibrational frequencies and the electronic properties of doped systems. The complex formation appears when the iodine concentration is greater than $12.5 \%$ and $6.25 \%$ for the graphene and bilayer doped respectively. Moreover, impurity bands below and above the Fermi level appear, corresponding to an effective hole doping of graphene equal to $1.1 \times 10^{13} \mathrm{~cm}^{-2}$. Additionally, we have studied the thermal stability of the adsorbed ions from ab initio molecular dynamics, carried out at $300 \mathrm{~K}$. At this temperature, the polyiodide complexes still exist, the adsorbed species lead to a power spectrum shift of the G-band, around $3 \mathrm{~cm}^{-1}$, indicating successful pdoping. Therefore, we can conclude that the high concentration or strong confinement of iodine molecule on a graphene layer generates iodide anions through a charge transfer process.

\section{Acknowledgments}

D. Tristant thanks the Midi-Pyrénées Région and the PRES Université de Toulouse for $\mathrm{PhD}$ funding. The authors also acknowledge the Calcul en Midi-Pyrénées initiative-CALMIP (Project p0812) for allocations of computer time. Part of this work was also performed using HPC resources from GENCIIDRIS (Project x2015096649). We would like to thank L. Alvarez and J.L. Bantignies for sharing their knowledge in iodine/carbon systems. We also thank Franck Jolibois for fruitful discussions. I. C. Gerber thanks CNRS for financial support.

\section{References}

1 A. N. Rudenko, F. J. Keil, M. I. Katsnelson and A. I. Lichtenstein, Phys. Rev. $B, 2010, \mathbf{8 2}, 035427$.

2 S. W. Chu, S. J. Baek, D. C. Kim, S. Seo, J. S. Kim and Y. W. Park, Synthetic Metals, 2012, 162, 1689-1693.

3 S. Ghosh, S. S. Yamijala, S. K. Pati and C. Rao, RSC Advances, 2012, 2, 1181-1188.

4 X. Luo, C. Fang, X. Li, W. Lai and T. Liang, Journal of Nuclear Materials, 2013, 441, $113-118$.

5 S. Y. Davydov and G. Sabirova, Technical Physics Letters, 2011, 37, 515518.

6 Z. Wang, W. Wang, M. Wang, X. Meng and J. Li, Journal of Materials Science, 2013, 48, 2284-2289.

7 L. D'Arsié, S. Esconjauregui, R. Weatherup, Y. Guo, S. Bhardwaj, A. Centeno, A. Zurutuza, C. Cepek and J. Robertson, Applied Physics Letters, 2014, 105, 103103.

8 X. Fan, L. Liu, J.-L. Kuo and Z. Shen, The Journal of Physical Chemistry $C, 2010,114,14939-14945$.

9 M. Ijäs, P. Havu and A. Harju, Phys. Rev. B, 2012, 85, 035440.

10 D. Tristant, P. Puech and I. C. Gerber, The Journal of Physical Chemistry C, 2015, 119, 12071-12078.

11 Z. Chen, P. Darancet, L. Wang, A. C. Crowther, Y. Gao, C. R. Dean, T. Taniguchi, K. Watanabe, J. Hone, C. A. Marianetti and L. E. Brus, ACS Nano, 2014, 8, 2943-2950.

12 A. Yaya, C. P. Ewels, I. Suarez-Martinez, P. Wagner, S. Lefrant, A. Okotrub, L. Bulusheva and P. R. Briddon, Phys. Rev. B, 2011, 83, 045411 .

13 H. L. Poh, P. Šimek, Z. Sofer and M. Pumera, Chemistry-A European Journal, 2013, 19, 2655-2662.

14 Z. Yao, H. Nie, Z. Yang, X. Zhou, Z. Liu and S. Huang, Chem. Commun., 2012, 48, 1027-1029.

15 Z. Wu, Y. Han, R. Huang, X. Chen, Y. Guo, Y. He, W. Li, Y. Cai and N. Wang, Nanoscale, 2014, 6, 13196-13202.

16 J. Vala, R. Kosloff and J. N. Harvey, The Journal of Chemical Physics, 2001, 114, 7413-7423.

17 N. Jung, N. Kim, S. Jockusch, N. J. Turro, P. Kim and L. Brus, Nano letters, $2009,9,4133-4137$.

18 G. Kalita, K. Wakita, M. Takahashi and M. Umeno, Journal of Materials Chemistry, 2011, 21, 15209-15213.

19 H. Kim, O. Renault, A. Tyurnina, J.-P. Simonato, D. Rouchon, D. Mariolle, N. Chevalier and J. Dijon, Applied Physics Letters, 2014, 105, 011605. 
20 X. Fan, E. C. Dickey, P. Eklund, K. Williams, L. Grigorian, R. Buczko, S. Pantelides and S. Pennycook, Physical review letters, 2000, 84, 46214624.

21 L. Grigorian, K. Williams, S. Fang, G. Sumanasekera, A. Loper, E. Dickey, S. Pennycook and P. Eklund, Physical review letters, 1998, 80, 5560.

22 N. Bendiab, R. Almairac, S. Rols, R. Aznar, J.-L. Sauvajol and I. Mirebeau, Physical Review B, 2004, 69, 195415.

23 W. Zhou, S. Xie, L. Sun, D. Tang, Y. Li, Z. Liu, L. Ci, X. Zou, G. Wang, P. Tan et al., Applied physics letters, 2002, 80, 2553-2555.

24 A. M. Rao, P. Eklund, S. Bandow, A. Thess and R. E. Smalley, Nature, 1997, 388, 257-259.

25 J. Cambedouzou, J.-L. Sauvajol, A. Rahmani, E. Flahaut, A. Peigney and C. Laurent, Phys. Rev. B, 2004, 69, 235422.

26 Y. Baskin and L. Meyer, Phys. Rev., 1955, 100, 544-544.

27 M. Lewin, Handbook of Fiber Chemistry, $3^{\text {rd }}$ ed. International Fiber Science and Technology, CRC Press, p. 944, 2006.

28 G. Kresse and J. Hafner, Phys. Rev. B, 1993, 47, 558-561.

29 G. Kresse and J. Hafner, Phys. Rev. B, 1994, 49, 14251-14269.

30 G. Kresse and J. Furthmüller, Phys. Rev. B, 1996, 54, 11169-11186.

31 G. Kresse and J. Furthmüller, Computational Materials Science, 1996, 6, $15-50$.

32 P. E. Blöchl, Physical Review B, 1994, 50, 17953.

33 C. L. Fu and K. M. Ho, Phys. Rev. B, 1983, 28, 5480-5486.

34 J. Klimeš, D. R. Bowler and A. Michaelides, Journal of Physics: Condensed Matter, 2010, 22, 022201.

35 J. c. v. Klimeš, D. R. Bowler and A. Michaelides, Phys. Rev. B, 2011, 83, 195131.

36 T. Hu and I. C. Gerber, The Journal of Physical Chemistry C, 2013, 117, 2411-2420.

37 T. Hu and I. C. Gerber, Chemical Physics Letters, 2014, 616, 75-80.

38 L. Kong, A. Enders, T. S. Rahman and P. A. Dowben, Journal of Physics: Condensed Matter, 2014, 26, 443001.

39 D. Tristant, Y. Wang, I. Gerber, M. Monthioux, A. Pénicaud and P. Puech, Journal of Applied Physics, 2015, 118, 044304.

40 A. Tkatchenko and M. Scheffler, Physical review letters, 2009, 102, 073005 .

41 T. Bučko, S. Lebègue, J. Hafner and J. Ángyán, Physical Review B, 2013, 87, 064110

42 P. E. Blöchl, O. Jepsen and O. K. Andersen, Phys. Rev. B, 1994, 49, 16223 16233.

43 W. Tang, E. Sanville and G. Henkelman, Journal of Physics: Condensed Matter, 2009, 21, 084204.

44 E. Sanville, S. D. Kenny, R. Smith and G. Henkelman, Journal of computational chemistry, 2007, 28, 899-908.
45 G. Henkelman, A. Arnaldsson and H. Jónsson, Computational Materials Science, 2006, 36, 354-360.

46 K. Momma and F. Izumi, Journal of Applied Crystallography, 2011, 44, $1272-1276$.

47 G. Li, A. Luican and E. Y. Andrei, Phys. Rev. Lett., 2009, 102, 176804.

48 S. Nosé, The Journal of Chemical Physics, 1984, 81, 511-519.

49 S. Nosé, Molecular physics, 1984, 52, 255-268.

50 G. J. Martyna, M. L. Klein and M. Tuckerman, The Journal of chemical physics, 1992, 97, 2635-2643.

51 K. Wendler, M. Brehm, F. Malberg, B. Kirchner and L. Delle Site, Journal of Chemical Theory and Computation, 2012, 8, 1570-1579.

52 P. Venezuela, M. Lazzeri and F. Mauri, Phys. Rev. B, 2011, 84, 035433.

53 P. Puech, T. Hu, A. Sapelkin, I. Gerber, V. Tishkova, E. Pavlenko, B. Levine, E. Flahaut and W. Bacsa, Phys. Rev. B, 2012, 85, 205412. 